\title{
Some results on the structure of multipoles in the study of snarks*
}

\author{
M. A. Fiol J. Vilaltella \\ Departament de Matemàtica Aplicada IV \\ Universitat Politècnica de Catalunya, BarcelonaTech \\ Barcelona, Catalonia \\ \{fiol, joan.vilaltella\}@ma4.upc.edu
}

Submitted: Aug 2, 2013; Accepted: Feb 4, 2015; Published: Feb 25, 2015

Mathematics Subject Classifications: 05C15, 05C05, 05C38

\begin{abstract}
Multipoles are the pieces we obtain by cutting some edges of a cubic graph in one or more points. As a result of the cut, a multipole $M$ has vertices attached to a dangling edge with one free end, and isolated edges with two free ends. We refer to such free ends as semiedges, and to isolated edges as free edges. Every 3 -edge-coloring of a multipole induces a coloring or state of its semiedges, which satisfies the Parity Lemma. Multipoles have been extensively used in the study of snarks, that is, cubic graphs which are not 3-edge-colorable. Some results on the states and structure of the so-called color complete and color closed multipoles are presented. In particular, we give lower and upper linear bounds on the minimum order of a color complete multipole, and compute its exact number of states. Given two multipoles $M_{1}$ and $M_{2}$ with the same number of semiedges, we say that $M_{1}$ is reducible to $M_{2}$ if the state set of $M_{2}$ is a non-empty subset of the state set of $M_{1}$ and $M_{2}$ has less vertices than $M_{1}$. The function $v(m)$ is defined as the maximum number of vertices of an irreducible multipole with $m$ semiedges. The exact values of $v(m)$ are only known for $m \leqslant 5$. We prove that tree and cycle multipoles are irreducible and, as a byproduct, that $v(m)$ has a linear lower bound.
\end{abstract}

Keywords: cubic graph, edge-coloring, snark, multipole, Parity Lemma, states, color complete, color closed, separable, irreducible, tree, cycle, linear recurrence

*Research supported by the Ministerio de Ciencia e Innovación, Spain, and the European Regional Development Fund under project MTM2011-28800-C02-01, and the Catalan Research Council under project 2009SGR1387. 


\section{Introduction}

A cubic graph can be subdivided by an edge cut into parts known as multipoles. As a result of the cut, a multipole has half-edges with one free end which we call semiedges. An isolated edge with both free ends can be viewed as a multipole, too. We call it a free edge, and we refer to its free ends as semiedges, too. A multipole need not be connected. We call $m$-pole a multipole with $m$ semiedges. According to the Parity Lemma, if a multipole can be 3-edge-colored then the number of semiedges of each color must have the same parity as $m$. Therefore, the possible colorings of the semiedges are not arbitrary. We refer to such colorings of the semiedges as states, where the ordering of the semiedges is fixed but the 3 colors are interchangeable. Multipoles and their states are used to study non-3-edge-colorable cubic graphs, called snarks. See, for instance, Isaacs [8], Gardner [6], Goldberg [7], and Fiol [3]. A multipole with all states permitted by the Parity Lemma is known as a color complete multipole, and a 3-edge-colorable multipole that has at least one state in common with any other 3-edge-colorable multipole with the same number of semiedges is known as a color closed multipole. Nedela and Škoviera [12] posed the question whether color closed non color complete multipoles exist for $m \geqslant 5$. Karabáš, Máčajová and Nedela [10] affirmatively answered this question, giving examples obtained by computer search. We also give examples for $m \in\{5,6\}$ and explain a method to obtain them. Moreover, we give other results on the states and structure of multipoles, in particular of color complete multipoles. Namely, we give an exact formula for the number of states of a color complete $m$-pole, we prove that color complete $m$-poles exist for every non-trivial value of $m$, and we give lower and upper bounds on the minimum order of a color complete $m$-pole as a function of $m$. This gives a partial solution to the problem of finding a multipole with a given set of states or feasibility problem (see [10]). With this same motivation in mind, we also give the minimum numbers of states of $m$-poles for $m \leqslant 6$, and we study the $m$-poles with the minimum possible number of vertices $(0$ if $m$ is even, and 1 if $m$ is odd) which we call minimal m-poles. Given two multipoles $M_{1}$ and $M_{2}$ with the same number of semiedges, we say that $M_{1}$ is reducible to $M_{2}$ if the state set of $M_{2}$ is a non-empty subset of the state set of $M_{1}$ and $M_{2}$ has less vertices than $M_{1}$. The function $v(m)$, introduced by Fiol [3], is defined as the maximum number of vertices of an irreducible $m$-pole. Only a few values of $v(m)$ are known. We prove that tree and cycle multipoles are irreducible and, as a byproduct, that $v(m)$ has a linear lower bound.

\section{Multipoles and Tait colorings}

A multipole or $m$-pole $M=(V, E, \mathcal{E})$ consists of a finite set of vertices $V$, a set of edges $E$ which are unordered pairs of vertices, and a set $\mathcal{E}$ whose $m$ elements $\epsilon_{1}, \ldots, \epsilon_{m}$ are called semiedges. We use the notation $V(M), E(M)$ and $\mathcal{E}(M)$ if we want to indicate that $V$, $E$ and $\mathcal{E}$ are, respectively, the vertex, edge and semiedge sets of a certain multipole $M$. Each semiedge is associated either with one vertex or with another semiedge making up what is usually called an isolated or free edge. For instance, Fig. 1(a) shows a 7-pole with two free edges. Notice that a multipole can be disconnected or even be 'empty', in the 
sense that it can have no vertices. The diagram of a generic $m$-pole will be as shown in Fig. 1(b).

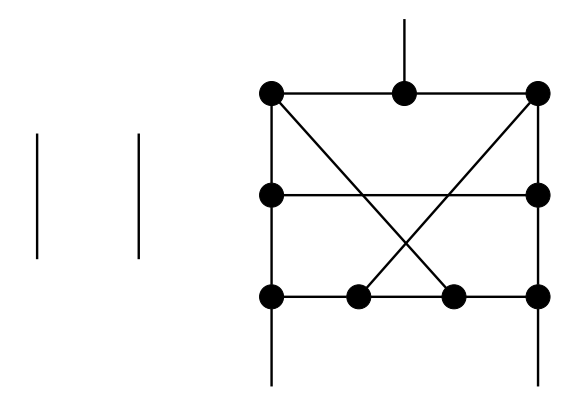

(a)

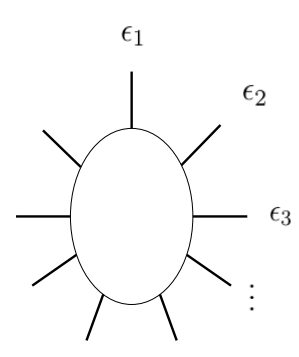

$(b)$

Figure 1: A 7-pole with two free edges $(a)$ and a generic multipole $(b)$.

In this paper we follow notation in $[3,4]$. For instance, if the semiedge $\epsilon$ is associated with vertex $u$, we say that $\epsilon$ is incident to $u$ and, following Goldberg's notation [7], we write $\epsilon=(u)$. Also, by joining the semiedges $(u)$ and $(v)$ we get the edge $(u, v)$. The degree of a vertex $u$, denoted by $\operatorname{deg}(u)$, is the number of edges plus the number of semiedges incident to it. In this paper, we only consider cubic multipoles, that is, those for which $\operatorname{deg}(u)=3$ for all $u \in V$. Thus, the simplest 2- and 3-poles are a free edge and a vertex with three incident semiedges. We denote them by $\boldsymbol{e}$ and $\boldsymbol{v}$, respectively.

Given a multipole $M$, we denote by $G[M]$ the graph obtained from $M$ by removing all its semiedges. Using this definition, we say that a multipole $N$ is contained in $M$, or that $N$ is a submultipole of $M$, when $G[N]$ is a subgraph of $G[M]$. Note that if $G[N]$ is a proper subgraph of $G[M]$ then $N$ can be obtained from $M$ by 'cutting' (in one or more points) some of its edges. The distance $\partial(\epsilon, \zeta)$ between two semiedges $\epsilon=(u)$ and $\zeta=(v)$ is defined to be the distance in $G[M]$ between its incident vertices $u$ and $v$.

When $G[M]$ is a tree or a forest we refer to $M$ as a tree multipole or a forest multipole respectively. Analogously, if $G[M]$ is a cycle we say that $M$ is a cycle multipole. A simple counting reasoning allows us to see that a tree $m$-pole has $n=m-2$ vertices, whereas a cycle $m$-pole has $n=m$ vertices. In general, for every $m$-pole with $n$ vertices, we have that $m \equiv n(\bmod 2)$ and the following result holds:

Lemma 2.1. Let $M$ be an m-pole with $n=|M|$ vertices and c components. Then,

$$
n \geqslant m-2 c,
$$

with equality if and only if $M$ is a forest multipole.

Proof. For $i=1, \ldots, c$, let $n^{(i)}$ and $m^{(i)}$ denote, respectively, the numbers of vertices and semiedges of the $i$-th component. Then the result follows from the fact that $n^{(i)} \geqslant m^{(i)}-2$ with equality if and only if the $i$-th component is a tree multipole. 
Let $C=\{1,2,3\}$ be a set of 'colors'. Then, a 3-edge-coloring or Tait coloring of an $m$-pole $(V, E, \mathcal{E})$ is a mapping $\phi: E \cup \mathcal{E} \rightarrow C=\{1,2,3\}$ such that all the edges and/or semiedges incident with a vertex receive distinct colors, and each isolated edge has both semiedges with the same color. For example, Fig. 2 shows a Tait coloring of a 6 -pole. Note that the numbers of semiedges with the same color have the same parity. The following basic lemma states that this is always the case.

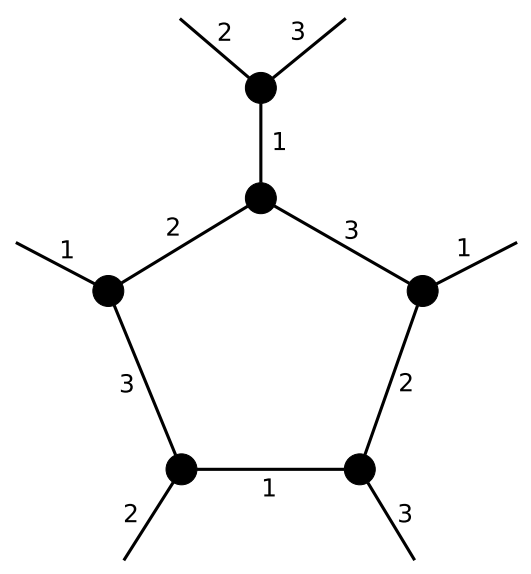

Figure 2: A Tait coloring of a 6-pole.

The Parity Lemma. Let $M$ be a Tait colored $m$-pole with $m_{i}$ semiedges having color $i=1,2,3$. Then,

$$
m_{1} \equiv m_{2} \equiv m_{3} \equiv m \quad(\bmod 2)
$$

This result has been used extensively in the construction of snarks (see, for instance, Isaacs [8], Goldberg [7], Chetwynd and Wilson [2], Watkins [16], Kochol [11] and Steffen $[13])$.

\section{Color completeness}

Given a multipole $M$ with semiedges $\epsilon_{1}, \ldots, \epsilon_{m}$, its set of semiedge colorings or states is

$$
\operatorname{Col}(M)=\left\{\left(\phi\left(\epsilon_{1}\right), \ldots, \phi\left(\epsilon_{m}\right)\right): \phi \text { is a Tait coloring of } M\right\} / \sim,
$$

where two states $S_{1}$ and $S_{2}$ are in the same equivalence class $\left(S_{1} \sim S_{2}\right)$ if they can be transformed into each other by a permutation of the colors.

A multipole $M$ is color complete if it has all the possible semiedge colorings allowed by the Parity Lemma. We call these states admissible. If a given multipole $M$ has a state $S$, we say that $S$ is realizable in $M$. Two multipoles $M_{1}$ and $M_{2}$ are color equivalent if $\operatorname{Col}\left(M_{1}\right)=\operatorname{Col}\left(M_{2}\right)$, that is, if each state of one multipole has an equivalent in the 
state set of the other. For instance, we consider the states $(1,1,2,2)$ and $(3,3,1,1)$ to be equivalent, and the multipoles with state sets $\{(1,2,3,1,1),(1,2,3,2,2),(1,2,3,3,3)\}$ and $\{(2,3,1,2,2),(2,3,1,3,3),(2,3,1,1,1)\}$ to be color equivalent. A state set is realized by the multipole $M$ if it is color equivalent to $\operatorname{Col}(M)$. Not surprisingly, the number of states $\sigma(m)=|\operatorname{Col}(M)|$ of a color complete $m$-pole $M$ coincides with the number of possible colorings of the planar regions in the $m$-ring of an almost triangulation [5, p. 190]. This is because of the equivalence, proved by Tait $[14,15]$, that the regions of a planar cubic graph $G$ are 4-colorable if and only if $G$ is 3-edge-colorable. Here we use the properties of 3-edge-colored multipoles to give a short derivation of the formula for such a number.

Proposition 3.1. Let $M$ be a color complete $m$-pole. Then, its number of states is

$$
\sigma(m)=\frac{1}{8}\left[3^{m-1}+2+(-1)^{m} 3\right] .
$$

Proof. We prove that $\sigma(m)$ satisfies the following recurrence:

$$
\sigma(m)=2 \sigma(m-1)+3 \sigma(m-2)-1
$$

with initial values $\sigma(2)=\sigma(3)=1$. Note that, given the semiedges $\epsilon_{1}$ and $\epsilon_{2}$, the set of states can be partitioned into two subsets: Those with $\phi\left(\epsilon_{1}\right)=\phi\left(\epsilon_{2}\right)$ and those with $\phi\left(\epsilon_{1}\right) \neq \phi\left(\epsilon_{2}\right)$ for some Tait coloring $\phi$. Here and henceforth, the letters $a, b, c$ stand for the colors 1,2,3 in any order, and ' $*$ ' denotes any color. The following facts give the recurrence:

(i) Every realizable state $(a, *,(m-2), *)$ of an $(m-1)$-multipole gives rise to two realizable states, $(b, c, *, \stackrel{(m-2)}{\cdots}, *)$, and $(c, b, *, \stackrel{(m-2)}{\cdots}, *)$, of an $m$-pole.

(ii) Every state $(a, *, \stackrel{(m-3)}{\cdots}, *)$ of an $(m-2)$-multipole gives rise to three realizable states, $(a, a, a, *, \stackrel{(m-3)}{\cdots}, *),(b, b, a, *, \stackrel{(m-3)}{\cdots}, *)$, and $(c, c, a, *,(m-3), *)$, of an $m$-pole.

(iii) If $m$ is odd, in $(i)$ the realizable state $(a, \stackrel{(m-1)}{\cdots}, a)$ gives two equivalent states. Similarly, if $m$ is even, in $(i i)$ the realizable state $(a, \stackrel{(m-2)}{\cdots}, a)$ gives two equivalent states (in both cases, up to permutation of colors $b$ and $c$ ).

Then, a particular solution of $(2)$ is $\sigma(m)=1 / 4$, whereas a general solution of the corresponding homogeneous equation turns out to be $\sigma(m)=\alpha 3^{m}+\beta(-1)^{m}$ where $\alpha, \beta \in$ $\mathbb{R}$. Adding up and imposing the initial conditions we obtain the result.

Color complete multipoles can be constructed recursively by using the following result:

Proposition 3.2. Let $M_{1}$ and $M_{2}$ be two color complete multipoles, with $m^{(1)}+r$ and $m^{(2)}+r$ semiedges respectively, where $m^{(1)}, m^{(2)} \geqslant 2, r \geqslant 2$. For $k=1, \ldots, r$, choose the semiedges $\left(u_{k}\right)$ from $M_{1}$ and $\left(v_{k}\right)$ from $M_{2}$ and join them to obtain the edges $\left(u_{k}, v_{k}\right)$. Then, the obtained multipole $M$, with $m=m^{(1)}+m^{(2)}$ semiedges, is also color complete. 
Proof. Let $S$ be a state of $M$, and for $j=1,2$ and $i=1,2,3$, let $m_{i}, r_{i}$, and $m_{i}^{(j)}$ be, respectively, the number of semiedges of $M$, the number of edges $\left(u_{k}, v_{k}\right)$ of $M$, and the number of semiedges of $M_{j}$, having color $i$. Then, $m^{(j)}=m_{1}^{(j)}+m_{2}^{(j)}+m_{3}^{(j)}$ and $r=r_{1}+r_{2}+r_{3}$ and, by the Parity Lemma, we must have:

$$
\begin{aligned}
m_{i}^{(1)}+m_{i}^{(2)} & \equiv m^{(1)}+m^{(2)} & & (\bmod 2), \\
m_{i}^{(1)}+r_{i} & \equiv m^{(1)}+r & & (\bmod 2), \\
m_{i}^{(2)}+r_{i} & \equiv m^{(2)}+r & & (\bmod 2) .
\end{aligned}
$$

Now, we claim that there exist states $S_{1}$ of $M_{1}$ and $S_{2}$ of $M_{2}$ inducing the state $S$ of $M$. In other words, given $m_{i}^{(1)}$ and $m_{i}^{(2)}$ satisfying (3), we want to find some values of $r_{i}$, $i=1,2,3$, satisfying (4) and (5). This gives

$$
\begin{aligned}
r_{i} \equiv m^{(1)}+r-m_{i}^{(1)} & (\bmod 2), \\
r_{i} \equiv m^{(2)}+r-m_{i}^{(2)} & (\bmod 2),
\end{aligned}
$$

which has a solution if and only if the two right hand terms have the same parity. That is,

$$
m^{(1)}-m_{i}^{(1)} \equiv m^{(2)}-m_{i}^{(2)} \quad(\bmod 2) .
$$

But this is precisely the condition (3). To see why the condition on $r$ is necessary, note first that the case $r=0$ is discarded by the observation that a color complete multipole must be connected: otherwise, the Parity Lemma should hold for every connected component, forbidding states that would be admissible as states of the entire multipole. Hence, if $r$ is even, then $r \geqslant 2$. Consider now the case $r=1$. We are assuming that $m^{(1)} \geqslant 2$. If $m^{(1)}+r$ is odd, the state with $m_{1}^{(1)} \equiv m_{2}^{(1)} \equiv m_{3}^{(1)} \equiv \alpha$, where $\alpha \in\{0,1\}$, imposes $r_{1} \equiv r_{2} \equiv r_{3} \equiv 1-\alpha$. Therefore, if $\alpha=0$ then $r_{1}, r_{2}, r_{3} \geqslant 1$, and if $\alpha=1$ then $r_{i} \geqslant 2$ for some $i \in\{1,2,3\}$. The case with even $m^{(1)}+r$ is similar.

As a consequence, we have the following result on the minimum order $n(m)$ of a color complete $m$-pole:

Theorem 3.3. For a given $m \geqslant 5$, the minimum number of vertices of a color complete $m$-pole satisfies the bounds:

(a) If $m$ is odd, then $m+2 \leqslant n(m) \leqslant 10 m-37$.

(b) If $m$ is even, then $m+2 \leqslant n(m) \leqslant 10 m-40$.

Proof. First we deal with the lower bounds. For $m \geqslant 5$, it is obvious that a complete $m$-pole cannot have two semiedges incident to the same vertex. This would preclude a common color for both semiedges, a possibility admitted by the Parity Lemma. We have seen that a color complete multipole must be connected. Moreover, if a connected multipole with no two semiedges at distance 0 has $m$ semiedges and only $m$ vertices, then it must be a cycle with a semiedge on each vertex, but such an $m$-pole cannot be complete either 
because certain states admissible by the Parity Lemma are not possible in a cycle $m$-pole with $m \geqslant 5$ (see Lemma 7.3). Also according to parity considerations, $m \equiv n(\bmod 2)$, so a multipole with $m$ semiedges cannot have $m+1$ vertices. Summarizing, if $m \geqslant 5$, then a complete $m$-pole must have at least $m+2$ vertices.

Now we deal with the upper bounds. Color complete multipoles can be constructed recursively as seen in Proposition 3.2, and specifically with $r=2$. Explicit color complete multipoles are known for $m=5$, Fig. 3(a), and $m=6$, Fig. 3(b), with 13 and 20 vertices respectively. This allows us to start the recursive construction of color complete multipoles with higher values of $m$. Let $M_{m}$ denote a color complete $m$-pole of minimum order, and $\left|V\left(M_{m}\right)\right|$ its number of vertices. Then, for $m \geqslant 7$,

$$
\left|V\left(M_{m}\right)\right|=\left|V\left(M_{(m-2)+6-4}\right)\right| \leqslant\left|V\left(M_{m-2}\right)\right|+\left|V\left(M_{6}\right)\right|=\left|V\left(M_{m-2}\right)\right|+20,
$$

and this gives $\left|V\left(M_{m}\right)\right| \leqslant 10 m-37$ for $m$ odd, and $\left|V\left(M_{m}\right)\right| \leqslant 10 m-40$ for $m$ even, as claimed.

Although we think that these bounds can be improved, we already see a linear behavior. As a corollary of the recursive construction, we conclude that color complete $m$-poles exist for any $m \geqslant 2$.

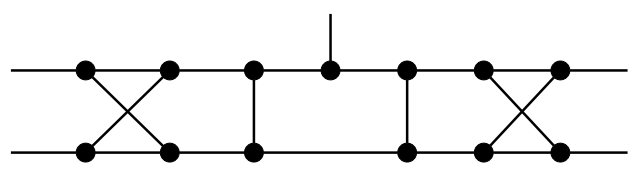

(a)

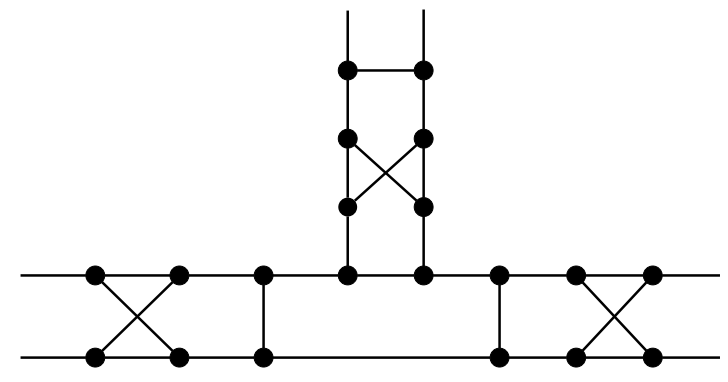

(b)

Figure 3: Color complete 5- and 6-poles.

\section{Color closedness}

Following the terminology of Nedela and Škoviera [12], we say that an $m$-pole $M$ is color closed if its state set has non empty intersection with the state set of any other Tait colorable $m$-pole $N$. Also in [12] a concept of snark irreducibility is defined, and proved equivalent to the fact that every nontrivial edge-cut, that is, one different from three edges incident with a vertex, separates the snark into Tait colorable components. Obviously, a color complete multipole is color closed, and if $m \geqslant 4$ then a color closed $m$-pole $M$ cannot be contained in an irreducible snark, because there is always at least one possible 
coloring of its $m$ semiedges that is in $\operatorname{Col}(M) \cap \operatorname{Col}(N)$ for any other Tait colorable $m$-pole $N$.

If $\mu(m)$ and $\sigma(m)$ are respectively the minimum and maximum number of states of an $m$-pole, then, by the pigeonhole principle, any $m$-pole with more than $\sigma(m)-\mu(m)$ states must be color closed. If it has less than $\sigma(m)$ states, then it is non-trivially color closed.

Proposition 4.1. The minimum number of states of Tait colorable 4-, 5-, and 6-poles are 2, 3, and 5 respectively.

Proof. We skip the easier cases of the 4- and 5-poles and explain in detail only the case of 6-poles.

Assume a 6-pole has state S. We may assume without loss of generality that either $S=(a, a, a, a, a, a), S=(a, a, a, a, b, b)$, or $S=(a, a, b, b, c, c)$. First we prove that a 6 -pole with the state $(a, a, a, a, a, a)$ has at least 5 states. Start a Kempe interchange of $a-b$ type at the first semiedge. We may assume that the resulting state is $(b, b, a, a, a, a)$. Alternatively, start a Kempe interchange of the same type at the third semiedge. Now we know that the Kempe chain cannot finish at the first or second semiedge. We may assume that the resulting state is $(a, a, b, b, a, a)$. Another possibility is to start the same type of Kempe interchange at the fifth semiedge. Now we know that it cannot finish at one of the first four semiedges. Hence, we assume that the resulting state is $(a, a, a, a, b, b)$. Summarizing, we can obtain at least three new states from $(a, a, a, a, a, a)$. Now take anyone of the three new states and start an arbitrary Kempe interchange of type $a-c$. This will give one new state, totalling five. The 6 -pole consisting of 3 free edges realizes this possibility. Therefore, $\mu(6) \leqslant 5$.

We must see that if a 6 -pole does not have the state $(a, a, a, a, a, a)$ then it cannot have less than 5 states. First assume that the 6-pole has at least one state with two colors, say $S_{1}=(a, a, a, a, b, b)$. Starting a Kempe interchange of type $a-b$ at the last semiedge we obtain one of the states $(b, a, a, a, b, a),(a, b, a, a, b, a),(a, a, b, a, b, a)$ or $(a, a, a, b, b, a)$, none of them equivalent to $S$. Assume it is $S_{2}=(a, a, a, b, b, a)$. Using arbitrary Kempe interchanges of type $a-c$ we can obtain two new three-colored non-equivalent states, one from $S_{1}$ and one from $S_{2}$. This gives a total of four states $\left\{S_{1}, S_{2}, S_{3}, S_{4}\right\}$. But taking $S_{1}$ and starting a Kempe interchange of type $a-b$ at the first semiedge we obtain one of the states $(b, b, a, a, b, b),(b, a, b, a, b, b),(b, a, a, b, b, b),(b, a, a, a, a, b)$ or $(b, a, a, a, b, a)$, the first three being equivalent to $(a, a, b, b, a, a),(a, b, a, b, a, a)$ and $(a, b, b, a, a, a)$. None has an equivalent state in $\left\{S_{1}, S_{2}, S_{3}, S_{4}\right\}$. Therefore, a fifth non-equivalent state $S_{5}$ exists.

Now assume that the 6-pole has only three-colored states, and that one of these states is $S_{1}=(a, a, b, b, c, c)$. Using Kempe interchanges and reasoning as in the previous paragraph, we may assume that the 6-pole has the states $S_{2}=(a, b, a, b, c, c)$, $S_{3}=(a, c, b, b, a, c)$ and $S_{4}=(a, a, b, c, b, c)$. All of them have repeated consecutive colors. But then a Kempe interchange of type $a-c$ starting at the first semiedge of $S_{4}$ gives either the state $(c, a, b, a, b, c)$ or $(c, a, b, c, b, a)$. None of them has repeated consecutive colors, and hence none of them has an equivalent state in $\left\{S_{1}, S_{2}, S_{3}, S_{4}\right\}$. Therefore, a fifth non-equivalent state $S_{5}$ exists. Summarizing, $\mu(6)=5$. 
The construction of color complete and color closed multipoles can be easily done by aggregation. In particular, the addition of the $X$-shaped 4-pole of Fig. $4(a)$ to an arbitrary $m$-pole by the junction of two pairs of semiedges is useful to increase its number of possible states (note the use of the $X$-shaped 4-pole in Section 3, Fig. 3). In this way, we have obtained the color closed multipoles of Figs. 4(b), 5(a), and $5(b)$, with 4, 5, and 6 semiedges respectively, and 3, 8, and 27 states respectively. The $X$-shaped 4 -pole is also color closed.

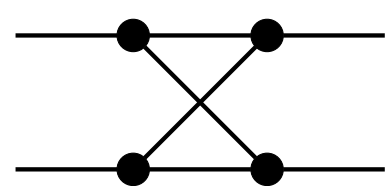

$(a)$

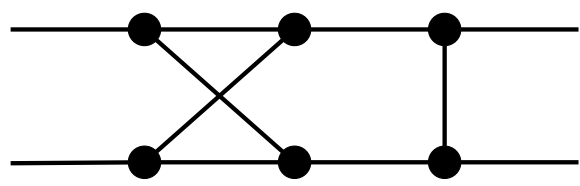

(b)

Figure 4: The $X$-shaped 4-pole and another color closed 4-pole.

In fact, color closed multipoles need not have a number of states close to the maximum, because many state sets are not realizable (that is, they are not realized by any multipole). In [10], color closed 6-poles with only 15 and 16 states are described, while the maximum number of states for 6 -poles is 31 .

The minimal multipoles are the $m$-poles with the minimum possible number of vertices. If $m$ is even, the minimal $m$-pole is constituted by $m / 2$ free edges $\boldsymbol{e}$ and it has no vertices. If $m$ is odd, the minimal $m$-pole is formed by the minimal 3 -pole $\boldsymbol{v}$, and $(m-3) / 2$ free edges.

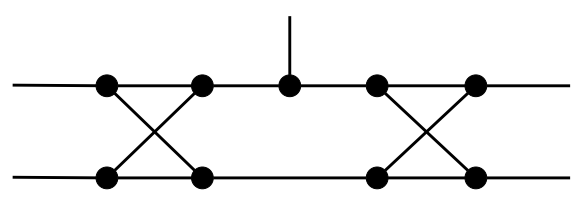

$(a)$

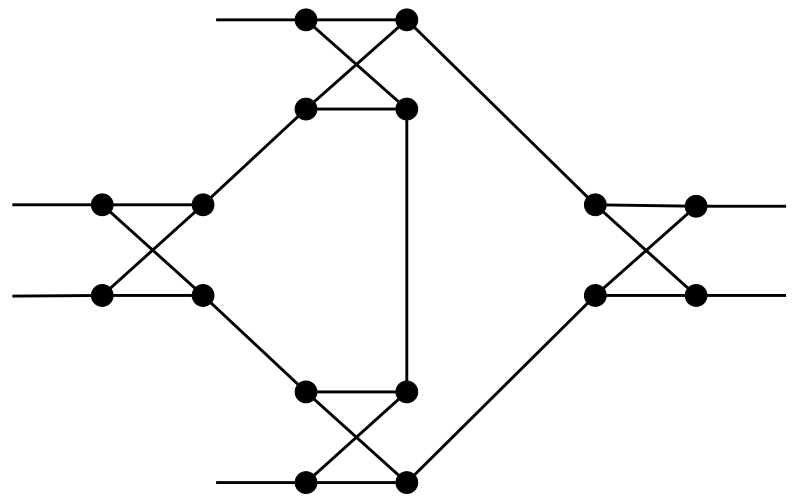

(b)

Figure 5: Color closed 5- and 6-poles. 
Theorem 4.2. Let $\rho(m)$ be the number of states of a minimal multipole.

(a) If $m$ is odd, then $\rho(m)=3^{\frac{m-3}{2}}$.

(b) If $m$ is even, then $\rho(m)=\frac{1}{2}\left(3^{\frac{m}{2}-1}+1\right)$.

Proof. (a) The minimal 3-pole $\boldsymbol{v}$ has a unique coloring, and each one of the $(m-3) / 2$ free edges has 3 possible colors.

(b) We have the following recurrence relation: $\rho(m+2)=3 \rho(m)-1$. To show this, consider $(m+2) / 2$ free edges. If the first $m / 2$ free edges have the same color, say $a$, then two of the three possible color assignments for the last free edge, $b$ and $c$, yield equivalent states because the colors are interchangeable. Otherwise, all color assignments for the last free edge yield non-equivalent states. Therefore, only two of the $3 \rho(\mathrm{m})$ possible states are equivalent to each other, and we must discard one of them. This gives the recurrence. The initial value is $\rho(2)=1$, corresponding to the unique state of a free edge. Using induction on $m$, or the same technique as in Section 3, we obtain the stated result.

From Proposition 3.1, Proposition 4.1, and Theorem 4.2, we see that the first values of $\mu(m), \rho(m)$, and $\sigma(m)$ are:

$$
\begin{array}{llrl}
\mu(4) & =\rho(4)=2, & \sigma(4) & =4, \\
\mu(5) & =\rho(5)=3, & \sigma(5) & =10, \\
\mu(6) & =\rho(6)=5, & \sigma(6) & =31 .
\end{array}
$$

From the results obtained, we conjecture that the minimum numbers of states are always attained by the minimal multipoles.

\section{$5 \quad$ Irreducibility}

For a given 3-edge-colorable $m$-pole $M$, let $\operatorname{Col}(M)$ denote the set of states of $M$ as defined in Section 3. Then, $M$ is said to be reducible when a 3-edge-colorable $m$-pole $N$ exists such that $|V(N)|<|V(M)|$ and $\operatorname{Col}(N) \subseteq \operatorname{Col}(M)$. Otherwise, we say that $M$ is irreducible. Obviously, all minimal multipoles are irreducible. If a multipole is not 3-edge-colorable, it can be further decomposed into smaller multipoles until all of them are, with the aim of studying the structure of snarks in terms of 3-edge-colorable multipoles and the possible ways of joining them.

The following result was first proved in [3]:

Proposition 5.1. Let $U$ be a snark. Then, for any integer $m \geqslant 1$ there exists a positive integer-valued function $v(m)$ such that any $m$-pole $M$ contained in $U$ with $|M|>v(m)$ is either not Tait colorable or reducible.

A reformulation of the same result was given later by Nedela and Škoviera [12, Th. 3.1]. In the same paper, the authors also gave credit to [3] for the introduction of the function $v(m)$, and rewrote it as $\kappa(k)$. 
The known values of $v(m)$ are $v(2)=0, v(3)=1$ (both are trivial results), $v(4)=2$ (Goldberg [7]), and $v(5)=5$ (Cameron, Chetwynd, and Watkins [1]). For $m=6$, Karabáš, Máčajová, and Nedela [10] proved that $v(6) \geqslant 12$.

The study of the function $v(m)$ is relevant in the study of the structure of snarks. More precisely, every value of $v(m)$ has a corresponding result for the decomposition of snarks. Moreover, according to a conjecture by Jaeger and Swart [9], every snark contains a cycle-separating edge-cut of size at most six. As commented in [10], if this were the case, then $v(6)$ would be the most interesting unknown value of $v(m)$.

The following basic lemma is simple but very useful in our study:

Lemma 5.2. Let $M$ be an irreducible multipole. Then, every submultipole $N \subset M$ is also irreducible.

Proof. We proceed by contradiction. If a submultipole $N$ of $M$ is reducible to, say, a multipole $N^{\prime}$, then $M$ is reducible to a multipole $M^{\prime}$ obtained replacing $N$ by $N^{\prime}$. This contradicts the fact that $M$ is irreducible.

As an example of application, note that, as every $m$-pole with $n \geqslant 1$ vertices contains an $(m+1)$-pole with $n-1$ vertices (just delete a semiedge $\epsilon=(v)$ and its incident vertex $v$ to create two new semiedges $\epsilon^{\prime}$ and $\left.\epsilon^{\prime \prime}\right)$, we have that $v(m+1) \geqslant v(m)-1$. Hence, from the results in [10], we know that $v(7) \geqslant 11$.

Note that $v(m)$ must have the same parity as $m$. We can be more precise with respect to the behavior of $v(m)$ if we use the following result.

Theorem 5.3. Let $M$ be an irreducible multipole. Then, the disjoint union of $M$ and one free edge $\boldsymbol{e}$ is also irreducible.

Proof. We proceed by contradiction. Assume that the disjoint union of $M$ and $\boldsymbol{e}$ is reducible to a submultipole $M^{\prime}$. We denote the semiedges of $\boldsymbol{e}$ by $\epsilon_{0}$ and $\epsilon_{1}$, and their corresponding semiedges in $M^{\prime}$ by $\epsilon_{0}^{\prime}$ and $\epsilon_{1}^{\prime}$. In the set of states of $M \cup \boldsymbol{e}$ (respectively $M^{\prime}$ ), all states must have $\phi\left(\epsilon_{0}\right)=\phi\left(\epsilon_{1}\right)$ (respectively $\phi\left(\epsilon_{0}^{\prime}\right)=\phi\left(\epsilon_{1}^{\prime}\right)$ ). This implies that $\epsilon_{0}^{\prime}$ and $\epsilon_{1}^{\prime}$ are not adjacent (that is, $\left.\partial\left(\epsilon_{0}^{\prime}, \epsilon_{1}^{\prime}\right)>0\right)$ unless they constitute a free edge $\boldsymbol{e}^{\prime}$ also in the submultipole $M^{\prime}$, but then $M$ would be trivially reducible to $M^{\prime}$ minus $\boldsymbol{e}^{\prime}$ and we would be done. Otherwise, we may join $\epsilon_{0}$ to $\epsilon_{1}$ in $M \cup \boldsymbol{e}$ and $\epsilon_{0}^{\prime}$ to $\epsilon_{1}^{\prime}$ in $M^{\prime}$. In the first case, we obtain $M$ and a trivial 'closed edge' which can be ignored. In the second case, we obtain a multipole $M^{\prime \prime}$ whose number of vertices is $\left|V\left(M^{\prime \prime}\right)\right|=\left|V\left(M^{\prime}\right)\right|<|V(M+\boldsymbol{e})|=|V(M)|$ and whose set of states is a subset of the set of states of $M$. By definition, $M$ would be reducible to $M^{\prime \prime}$, contradicting our assumption.

As a consequence, we have that $v(m) \geqslant v(m-2)$. Therefore, the function $v(m)$ is, at least, partially monotone in the sense that its even (respectively odd) values behave monotonically. 


\section{Trees and forests}

The number of states of a tree $m$-pole $T_{m}$, denoted by $t(m)=\left|\operatorname{Col}\left(T_{m}\right)\right|$, is easy to compute. Tree multipoles can be constructed recursively by the addition of vertices. A 3 -pole with a single vertex has only one possible state. Then, $t(3)=\left|\operatorname{Col}\left(T_{3}\right)\right|=1$. Every new vertex increases $m$ one unit and doubles the number of possible states. Then, $t(m)=2 t(m-1)$. As a consequence, $t(m)=2^{m-3}$.

An $m$-pole $M$ with $c$ components is called separable if there exists some $m$-pole $N$, with at least $c+1$ components, $|V(N)|=|V(M)|$, and $\operatorname{Col}(N) \subseteq \operatorname{Col}(M)$.

Now, we have the following result.

Theorem 6.1. Every tree multipole $T_{m}$ is non-separable and irreducible.

Proof. The proof is by induction. The result is trivial for $m=2,3$ since $T_{2}=\boldsymbol{e}$ and $T_{3}=\boldsymbol{v}$. Then, assume that the results holds for some $T_{m}$ with $m>3$. For some semiedge $(u)$, consider the $(m+1)$-pole $M$ obtained by joining one semiedge of $\boldsymbol{v}$ to $u$, so 'turning' (u) into two new semiedges $\epsilon_{1}=(v)_{1}$ and $\epsilon_{2}=(v)_{2}$. We first prove that $M$ is irreducible. Otherwise, there would exist an $(m+1)$-pole $M^{\prime}$ with $\left|V\left(M^{\prime}\right)\right|<|V(M)|=m-1$ (and hence $\left.\left|V\left(M^{\prime}\right)\right| \leqslant m-3\right)$ and $\operatorname{Col}\left(M^{\prime}\right) \subseteq \operatorname{Col}(M)$. Thus, $M^{\prime}$ must have at least two connected components, say, $M_{1}^{\prime}$ and $M_{2}^{\prime}$. Moreover, since $\epsilon_{1}$ and $\epsilon_{2}$ must have different colors in any Tait coloring of $M$, the same is true for the corresponding semiedges $\epsilon_{1}^{\prime}$ and $\epsilon_{2}^{\prime}$ in $M^{\prime}$. Consequently, such semiedges cannot be in different components of $M^{\prime}$. Without loss of generality, assume that $\epsilon_{1}^{\prime}, \epsilon_{2}^{\prime} \in \mathcal{E}\left(M_{1}^{\prime}\right)$. Now by joining $\epsilon_{1}^{\prime}$ and $\epsilon_{2}^{\prime}$ to two semiedges of $\boldsymbol{v}$, and doing the same operation to $\epsilon_{1}$ and $\epsilon_{2}$, so creating a digon, we respectively obtain the multipoles $N^{\prime}$ and $N$ with the following properties:

- $\operatorname{Col}\left(N^{\prime}\right) \subseteq \operatorname{Col}(N)=\operatorname{Col}\left(T_{m}\right)$ (as the created digon 2-pole in $N$ is color equivalent to $\boldsymbol{e})$.

- $\left|V\left(N^{\prime}\right)\right| \leqslant m-2=\left|V\left(T_{m}\right)\right|$.

Thus, if $\left|V\left(N^{\prime}\right)\right|<m-2, T_{m}$ would be reducible, and if $\left|V\left(N^{\prime}\right)\right|=m-2, T_{m}$ would be separable. In any case, we get a contradiction.

To prove that $M$ is also non-separable we again assume the contrary and iterate the above procedure until $M$ is proved to be color equivalent to some small tree multipole $T$, whereas $M^{\prime}$ is color equivalent to a multipole $N$ constituted by some isolated edges. But this again contradicts the fact that $\operatorname{Col}\left(M^{\prime}\right) \subseteq \operatorname{Col}(M)$.

As every tree $m$-pole $T_{m}$ has $m-2$ vertices, the above theorem allows us to state that $v(m) \geqslant m-2$ for $m \geqslant 2$.

Using basically the same proof as above, we have the following:

Proposition 6.2. Every forest multipole is non-separable and irreducible.

The following result shows that the number of states of a forest $m$-pole depends only on $m$ and its number $n$ of vertices. Let $f(n, m)$ denote the number of states of a forest $m$-pole $M$ with $n$ vertices. 
Lemma 6.3. If $n \leqslant m-4$, the following recurrence relation holds:

$$
f(n, m)=f(n, m-2)+f(n+1, m-1),
$$

with initial values $f(0,2)=1$ and $f(n, n+2)=2^{n-1}$ for $n>0$.

Proof. First notice that, if $n=m-2>0$ then a forest $m$-pole with $n$ vertices is in fact a tree $m$-pole, with number of states $2^{m-3}$ or $2^{n-1}$. If $n=0$ and $m=2$, then we have an isolated edge with only one state. This gives the starting values. If $n \leqslant m-4$, a forest $m$-pole with $n$ vertices is not a tree and has at least two components. Then, given two semiedges $\epsilon_{1}$ and $\epsilon_{2}$ (not in the same component), its set of states can be partitioned into two subsets: Those with $\phi\left(\epsilon_{1}\right)=\phi\left(\epsilon_{2}\right)$, and those with $\phi\left(\epsilon_{1}\right) \neq \phi\left(\epsilon_{2}\right)$, for some Tait coloring $\phi$ of $M$.

Case $\phi\left(\epsilon_{1}\right)=\phi\left(\epsilon_{2}\right)$ : The two semiedges can be joined and we obtain an $m^{\prime}$-pole $M^{\prime}$ with $m^{\prime}=m-2$ semiedges and $n^{\prime}=n$ vertices. As a consequence of $n \leqslant m-4$, the relation $n^{\prime} \leqslant m^{\prime}-2$ holds. Therefore, its number of states is $f\left(n^{\prime}, m^{\prime}\right)=f(n, m-2)$.

Case $\phi\left(\epsilon_{1}\right) \neq \phi\left(\epsilon_{2}\right)$ : The two semiedges can be joined to two semiedges $\epsilon_{1}^{\prime \prime}$ and $\epsilon_{2}^{\prime \prime}$ of a minimal 3-pole $\boldsymbol{v}$. Let $\epsilon_{3}^{\prime \prime}$ denote the remaining semiedge of $\boldsymbol{v}$. The result of the junction is a new $m^{\prime \prime}$-pole $M^{\prime \prime}$ with $m^{\prime \prime}=m-1$ semiedges, now including $\epsilon_{3}^{\prime \prime}$, and $n^{\prime \prime}=n+1$ vertices. Again, the relation $n^{\prime \prime} \leqslant m^{\prime \prime}-2$ holds as a consequence of $n \leqslant m-4$. Therefore, the number of states of $M^{\prime \prime}$ is $f\left(n^{\prime \prime}, m^{\prime \prime}\right)=f(n+1, m-1)$.

Finally, adding up all states we obtain the recurrence relation.

By means of a straightforward calculation, it can be proved that, if $0 \leqslant 2 k \leqslant m-2$ and $n \leqslant m-2(k+1)$, then the following formula holds:

$$
f(n, m)=\sum_{i=0}^{k} f(n+i, m-2 k+i)\left(\begin{array}{c}
k \\
i
\end{array}\right) .
$$

As a consequence, $f(n, m)$ can be easily calculated.

\section{Theorem 6.4.}

(a) The number of states of a forest $m$-pole with 0 vertices is:

$$
f(0, m)=\frac{1}{2}\left(3^{\frac{m-2}{2}}+1\right) .
$$

(b) The number of states of a forest $m$-pole with $n \geqslant 1$ vertices is:

$$
f(n, m)=2^{n-1} 3^{\frac{m-n}{2}-1} .
$$


Proof. (a) Assuming $n=0$ and $m=2 k+2$ in (6), and using the initial values, we have:

$$
\begin{aligned}
f(0, m) & =f(0,2 k+2)=\sum_{i=0}^{k} f(i, i+2)\left(\begin{array}{l}
k \\
i
\end{array}\right)=f(0,2)+\sum_{i=1}^{k} f(i, i+2)\left(\begin{array}{l}
k \\
i
\end{array}\right) \\
& =1+\sum_{i=1}^{k} 2^{i-1}\left(\begin{array}{l}
k \\
i
\end{array}\right)=1+\frac{1}{2} \sum_{i=1}^{k} 2^{i}\left(\begin{array}{l}
k \\
i
\end{array}\right) \\
& =1+\frac{1}{2}\left(3^{k}-1\right)=\frac{1}{2}\left(3^{k}+1\right)=\frac{1}{2}\left(3^{\frac{m-2}{2}}+1\right) .
\end{aligned}
$$

(b) Assuming $n \geqslant 1$ and $m=n+2 k+2$ in (6), and using the initial values, we have:

$$
\begin{aligned}
f(n, m) & =f(n, n+2 k+2)=\sum_{i=0}^{k} f(n+i, n+i+2)\left(\begin{array}{l}
k \\
i
\end{array}\right) \\
& =\sum_{i=0}^{k} 2^{n+i-1}\left(\begin{array}{c}
k \\
i
\end{array}\right)=2^{n-1} \sum_{i=0}^{k} 2^{i}\left(\begin{array}{c}
k \\
i
\end{array}\right)=2^{n-1} 3^{k}=2^{n-1} 3^{\frac{m-n}{2}-1} .
\end{aligned}
$$

Figure 6 shows the values of $f(m, n)$ for $2 \leqslant m \leqslant 14$ and $0 \leqslant n \leqslant 12$, and also the numbers of states of cycle $m$-poles, for which $n=m$.

The following result, consequence of Theorem 6.4, implies that the minimum number of states of a forest $m$-pole $M$ is attained when $M$ is a minimal $m$-pole. Note that in both cases we obtain $\rho(m)$.

Proposition 6.5. For a fixed even value of $m$, the minimum of $f(n, m)$ is $\frac{1}{2}\left(3^{\frac{m-2}{2}}+1\right)$, and for a fixed odd value of $m$, the minimum of $f(n, m)$ is $3^{\frac{m-3}{2}}$.

The set of states of a tree $m$-pole with $n$ vertices can be obtained algorithmically in the following way. Pick an arbitrary vertex $v$ with at least one incident semiedge $\epsilon$. Assume, without loss of generality, that $\epsilon$ has color $a$ and the remaining two edges or semiedges have color $b$ and $c$. The semiedge $\epsilon$ can be ignored and the rest of the tree can be viewed as a binary tree with $v$ as the root. At each one of the $n-1$ remaining vertices a binary coloring decision can be made, and each one of the $2^{n-1}$ corresponding binary sequences gives one possible state.

\section{Cycles}

In this section we prove the irreducibility of every cycle multipole for $m \geqslant 5$, and calculate the number of states of an arbitrary cycle multipole with $m \geqslant 1$.

Proposition 7.1. The number $c(m)$ of states of a cycle $m$-pole $C_{m}$ with $m \geqslant 1$ is

$$
c(m)=\frac{1}{3}\left(2^{m-1}+(-1)^{m}\right) .
$$


$m$

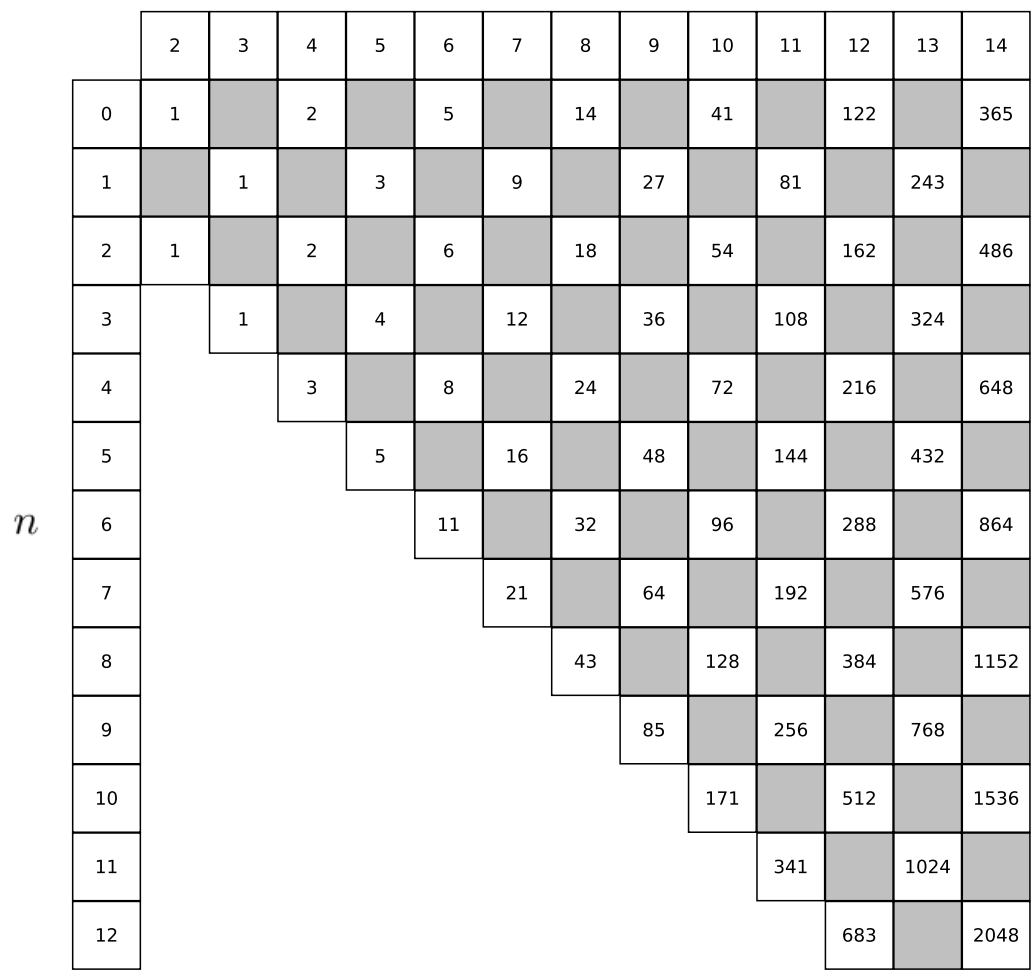

Figure 6: Numbers of states of forest $m$-poles with $n$ vertices. The rows $n=0$ and $n=1$ correspond to minimal $m$-poles, and the diagonal $n=m-2$ corresponds to tree $m$-poles. The numbers of states of cycle $m$-poles, corresponding to the diagonal $n=m$, are also represented.

Proof. The coloring of the semiedges is determined by the coloring of the edges of the cycle. Then, the number $c(m)$ verifies the following recurrence:

$$
c(m)=c(m-1)+2 c(m-2) .
$$

To show this, consider one arbitrary edge of the cycle, and the two edges adjacent to it. If these two edges have different color, the color of the middle edge is forced. If they have the same color, there are two possibilities for the color of the middle edge. In the first case, we delete the middle edge and make its two adjacent edges adjacent to each other: the resulting cycle has $m-1$ edges and $c(m-1) 3$-edge-colorings. In the second case, we delete the middle edge and identify its two adjacent edges as if they were one: the resulting cycle has $m-2$ edges and $c(m-2) 3$-edge-colorings, but in this case the deleted edge had two possible colors. This gives the recurrence for $c(m)$. Its initial values are $c(1)=0$ and $c(2)=1$. Using induction on $m$, or the same technique as in Section 3, we can prove the result. 
The set of states of a cycle $m$-pole can be obtained algorithmically in the following way. Cut an arbitrary edge $(u, v)$ of the cycle to obtain a tree $(m+2)$-pole, retaining the condition that the resulting semiedges $(u)_{1}$ and $(v)_{1}$ must have the same color. Assume, without loss of generality, that they have color $a$. As a result of the cut, $u$ and $v$ are at opposite ends of the tree. Ignore for the moment $v$, its nearest vertex and their incident semiedges. The result is a tree $m$-pole whose set of states can be determined as in the previous section, picking $u$ as the root and ignoring $(u)_{1}$ to obtain a binary tree. Now it is straightforward to extend the states of the partial tree $m$-pole to the states of the whole tree $(m+2)$-pole, with the condition that $(v)_{1}$ has color $a$ to match the color of $(u)_{1}$. This is an alternative way to obtain the enumeration result on the states of cycle multipoles.

Now we show that, as the tree multipoles, cycle multipoles are also irreducible. To this end, we need some previous lemmas.

Lemma 7.2. Let $\epsilon_{1}, \epsilon_{2}, \epsilon_{3}$ be three (ordered) semiedges of a m-tree multipole $T_{m}$ with $m \geqslant 3$. Then, the state $\left(\ldots, \epsilon_{1}, \epsilon_{2}, \epsilon_{3}, \ldots\right)=(\ldots, a, b, a, \ldots)$ is realizable unless one of the following conditions holds:

(i) $\partial\left(\epsilon_{1}, \epsilon_{3}\right)=0$.

(ii) $\partial\left(\epsilon_{1}, \epsilon_{2}\right)=\partial\left(\epsilon_{2}, \epsilon_{3}\right)=1$.

Proof. Let $\epsilon_{i}=\left(v_{i}\right), i=1,2,3$. First note that, as $\epsilon_{1}$ and $\epsilon_{2}$ are distinct, a coloring of the form $\left(\ldots, \epsilon_{1}, \epsilon_{2}, \epsilon_{3}, \ldots\right)=(\ldots, a, b, *, \ldots)$ is always possible. Then, the only cases where color $*$ cannot be $a$ are $(i)$ (trivially since then $v_{1}=v_{3}$ ) and (ii) since, then, edge $v_{1} v_{2}$ must have color $c$ and, hence, edge $v_{2} v_{3}$ must have color $a$ which is forbidden.

Lemma 7.3. Let $\epsilon_{i}, \epsilon_{i+1}, \ldots, \epsilon_{i+r-1}$, be $r$ successive semiedges of a m-cycle multipole $C_{m}$ with $r \geqslant m$ (subindexes understood modulo $m$ ). Then, the following states are not realizable:

(i) For $r=3,\left(\ldots, \epsilon_{i}, \epsilon_{i+1}, \epsilon_{i+2}, \ldots\right)=(\ldots, a, b, a, \ldots)$.

(ii) For $r=4,\left(\ldots, \epsilon_{i}, \epsilon_{i+1}, \epsilon_{i+2}, \epsilon_{i+3}, \ldots\right)=(\ldots, a, b, b, c, \ldots)$.

Proof. Let $\epsilon_{i+j}=\left(v_{i+j}\right), j=0, \ldots, 3$. Case $(i)$ is proved as in Lemma 7.2. Case $(i i)$ follows also easily by considering the two different possible colors, $a$ and $c$, of edge $v_{i+1} v_{i+2}$ and concluding that both lead to a contradiction.

Theorem 7.4. Every cycle multipole $C_{m}$ with $m \geqslant 5$ is irreducible.

Proof. The proof is by contradiction. Assume that $C_{m}$ is reducible to an $m$-pole $T$. As $|T| \leqslant m-2, T$ is either a tree multipole or it has at least two components. Since $\operatorname{Col}(T) \subseteq \operatorname{Col}\left(C_{m}\right)$, there must be a cyclic ordering $\left(\epsilon_{0}, \ldots, \epsilon_{m-1}, \epsilon_{0}, \ldots\right)$ of the semiedges of $T$, corresponding to the successive semiedges of $C_{m}$, such that every state of $T$ is also realizable in $C_{m}$. However, we will prove that, no matter how we choose such an ordering, this is not the case. We consider three possibilities: 
(a) $T$ is a tree.

(a1) Assume all the semiedges of $T$ come into pairs (that is, every pair of them is incident with a given vertex). Without loss of generality, suppose that $\epsilon_{i}$ and $\epsilon_{i+1}$ are incident to $u_{i}$ for $i=0,2, \ldots,(m-2) / 2$. Then, by Lemma 7.2 , in the cyclic ordering and in order to avoid the states $(\ldots, a, b, a, \ldots)$ we are forced to take $\left(\ldots, \epsilon_{1}, \epsilon_{j}, \epsilon_{2}, \epsilon_{j+1}, \ldots\right)$ with $j \geqslant 3$. But every choice for the semiedge $\epsilon_{k}$ next to $\epsilon_{j+1}$ allows the state $(\ldots, a, b, a, \ldots)$ for the semiedges $\left(\ldots, \epsilon_{2}, \epsilon_{j+1}, \epsilon_{k}, \ldots\right)$, which is not realizable in $C_{m}$ by Lemma 7.3.

(a2) Otherwise, $T$ must have some semiedge $\zeta$ at nonzero distance from every other semiedge, and also some pair $\left(\epsilon_{i}, \epsilon_{i+1}\right)$ of incident semiedges. Thus, at some point of the cyclic ordering, we must have $\left(\ldots, \zeta, \epsilon_{j}, \epsilon_{k}, \ldots\right)$ where $j \in\{i, i+1\}$. Then, again, all possible choices of $\epsilon_{k}$ allow the state $(\ldots, a, b, a, \ldots)$.

(b) $T$ has two components $X, Y$ : Let $X$ and $Y$ have semiedges $\epsilon_{i}$ and $\zeta_{j}$, respectively. Notice that either $X$ or $Y$ has at least three semiedges. Then, at some point of the cyclic ordering, we must go from, say, $\epsilon_{1} \in \mathcal{E}(X)$ to $\zeta_{2} \in \mathcal{E}(Y)$. Up to symmetries, there are three possibilities for the next semiedges to $\epsilon_{1}$ and $\zeta_{2}$ (see Fig. 7 where 'cyclic adjacency' between semiedges is represented by dotted lines). With no loss on clarity, we may omit the trailing periods in the notation of states:

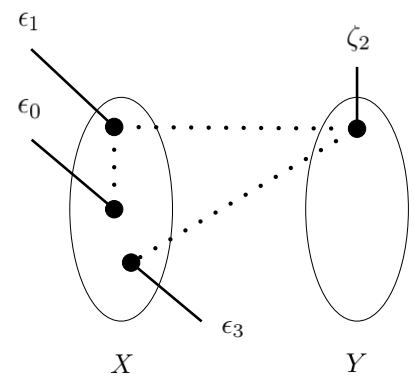

(i)

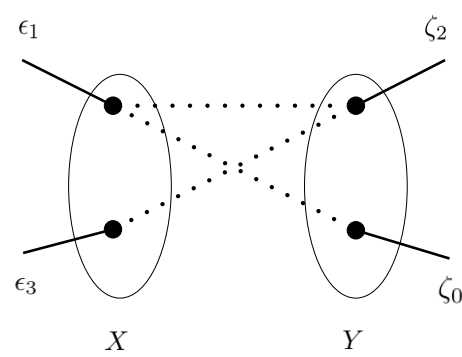

(ii)

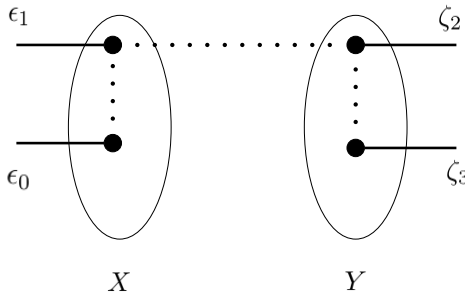

(iii)

Figure 7: Possible successions of semiedges.

(b1) Case $\left(\ldots, \epsilon_{0}, \epsilon_{1}, \zeta_{2}, \epsilon_{3}, \ldots\right)$ : Here, for any Tait coloring $\phi$ of $T$, and in order to avoid the state $(a, b, a)$ for the semiedge triples $\left(\epsilon_{0}, \epsilon_{1}, \zeta_{2}\right)$ and $\left(\epsilon_{1}, \zeta_{2}, \epsilon_{3}\right)$, it must be $\phi\left(\epsilon_{0}\right)=\phi\left(\epsilon_{1}\right)$ and $\phi\left(\epsilon_{1}\right) \neq \phi\left(\epsilon_{3}\right)$. Thus, the state of the successive semiedges must be $(a, a, *, b)$. Now consider the $a-b$ Kempe chain in $X$, starting from $\epsilon_{3}$ and interchange its colors to get the new Tait coloring $\phi^{\prime}$. If it ends to a semiedge different from $\epsilon_{0}, \epsilon_{1}$ we get the new state $(a, a, *, a)$. Otherwise, if it ends to $\epsilon_{0}$ or $\epsilon_{1}$ we get $(b, a, *, a)$ or $(a, b, *, a)$ respectively. In all cases we can adequately chose the color $*$ to get the state $(a, b, a)$ for three successive semiedges. 
(b2) Case $\left(\ldots, \zeta_{0}, \epsilon_{1}, \zeta_{2}, \epsilon_{3}, \ldots\right)$ : To avoid the state $(a, b, a)$ for the two semiedge triples it must be $\phi\left(\zeta_{0}\right) \neq \phi\left(\zeta_{2}\right)$ and $\phi\left(\epsilon_{1}\right) \neq \phi\left(\epsilon_{3}\right)$. Thus, the state of the successive semiedges can be chosen to be $(a, a, b, b)$. Let us consider the different possibilities for the semiedge following $\epsilon_{3}$. If this is $\epsilon_{4} \in \mathcal{E}(X)$, the semiedges $\left(\epsilon_{1}, \zeta_{2}, \epsilon_{3}, \epsilon_{4}\right)$ follow the pattern as in $(i)$, which has already been dealt with. Alternatively, if such a semiedge is $\zeta_{4} \in \mathcal{E}(Y)$, it cannot have color $c$ because of Lemma 7.3(ii). Thus, suppose that $\zeta_{4}$ has a color different from $c$, say $a$, and consider the $a-b$ Kempe chain in $Y$ starting from $\zeta_{4}$ and interchange its colors. Then, reasoning as above, we obtain the realizable state $(a, b, a)$ for three successive semiedges.

(b3) Case $\left(\ldots, \epsilon_{0}, \epsilon_{1}, \zeta_{2}, \zeta_{3}, \ldots\right)$ : Now, to avoid the state $(a, b, a)$ for the two semiedge triples it must be $\phi\left(\epsilon_{0}\right)=\phi\left(\epsilon_{1}\right)$ and $\phi\left(\zeta_{2}\right)=\phi\left(\zeta_{3}\right)$, so that we can chose the state of the successive semiedges to be $(a, a, b, b)$. Thus, the semiedge following $\zeta_{3}$ cannot have color $c$ because of Lemma 7.3(ii). For the situation of such a semiedge, we consider again two possibilities: If $\zeta_{4} \in Y$ and it has color $a$, the interchanging of color in the $a-c$ Kempe chain leads again to the state $(a, a, b, b, c)$. Otherwise, if $\zeta_{4}$ has color $b$, the $b$ - $c$ Kempe chain leads again to either such a state or to the color $c$ for some of the semiedges $\zeta_{2}$ or $\zeta_{3}$. But, again, both alternatives led to a realizable state $(c, b, c)$ (changing the color of $\epsilon_{1}$ from $a$ to $c$, if necessary). Finally, if $\epsilon_{4} \in X$ and it has colors $a$ or $b$, we reason as above with the respective $a-c$ or $b$ - $c$ Kempe chains to reach the states of type $(a, a, b, b, c)$ or $(a, b, a)$.

(c) $T$ has at least three components $X, Y, Z$ : Denote the semiedges of $X$ and $Y$ as above and let $Z$ have semiedges $\eta_{k}$. Note that in the cyclic ordering, and to avoid the state $(a, b, a)$, we cannot have 3 successive semiedges in different components $X, Y, Z$. Then, the only possibility to be considered, being different from those in $(b)$, is $\left(\epsilon_{0}, \zeta_{1}, \zeta_{2}, \eta_{3}\right)$. Then it must be $\phi\left(\zeta_{1}\right)=\phi\left(\zeta_{2}\right)$ and the corresponding state can be chosen to be $(a, b, b, c)$. But this is one of the non-desired states of Lemma 7.3.

Summarizing, $T$ has always a state not realizable in $C_{m}$. But this is in contradiction with our assumption. Thus, we conclude that $C_{m}$ is irreducible.

As every cycle $m$-pole $C_{m}$ has $m$ vertices, the above proposition allows us to state that if $m \geqslant 5$ then $v(m) \geqslant m$.

\section{Conclusions}

We calculated the exact number of states of tree, cycle, minimal and color complete multipoles with $m$ semiedges, and proved that color complete multipoles exist for every non trivial value of $m$. The minimum order of a color complete multipole has lower and upper linear bounds in terms of $m$. Moreover, we proved that results on the behavior of the function $v(m)$, which is only partially known but relevant in the study of the structure 
of snarks, can be obtained by the analysis of reducibility of multipoles, in particular tree and cycle multipoles.

\section{Acknowledgements}

The authors sincerely thank the anonymous referees for their helpful comments and suggestions.

\section{References}

[1] P. J. Cameron, A. G. Chetwynd and J. J. Watkins, Decomposition of snarks, J. Graph Theory 11 (1987), no. 1, 13-19.

[2] A. G. Chetwynd and R. J. Wilson, Snarks and supersnarks, in The Theory and Applications of Graphs (G. Chartrand, Y. Alavi, D. L. Goldsmith, L. Lesniak-Foster and D. R. Lick, eds.), John Wiley \& Sons, New York (1981), 215-241.

[3] M. A. Fiol, A Boolean algebra approach to the construction of snarks, in Graph Theory, Combinatorics and Applications, vol. 1 (Y. Alavi, G. Chartrand, O. R. Oellermann, and A. J. Schwenk, eds.), John Wiley \& Sons, New York (1991), 493-524.

[4] M. A. Fiol and J. Vilaltella, A simple and fast heuristic algorithm for edge-coloring of graphs, AKCE Int. J. Graphs Comb. 10 (2013), no. 3, 263-272.

[5] R. Fritsch and G. Fritsch, The Four-Color Theorem: History, Topological Foundations, and Idea of Proof, translated by J. Peschke, Springer (1998).

[6] M. Gardner, Mathematical Games: Snarks, Boojums and other conjectures related to the four-color-map theorem, Scientific American 234 (1976), no. 4, 126-130.

[7] M. K. Goldberg, Construction of class 2 graphs with maximum vertex degree 3, J. Combin. Theory Ser. B 31 (1981), no. 3, 282-291.

[8] R. Isaacs, Infinite families of nontrivial trivalent graphs which are not Tait colorable, Amer. Math. Monthly 82 (1975), no. 3, 221-239.

[9] F. Jaeger and T. Swart, Conjecture 1 and 2, in Combinatorics 79. Part II (M. Deza, I. G. Rosenberg, eds.), Annals of Discrete Mathematics 9 (1980), North-Holland, Amsterdam, p. 305, Problem Session.

[10] J. Karabáš, E. Máčajová, and R. Nedela, 6-decomposition of snarks, European J. Combin. 34 (2013), no. 1, 111-122.

[11] M. Kochol, Snarks without small cycles, J. Combin. Theory Ser. B 67 (1996), no. 1, $34-47$.

[12] R. Nedela and M. Škoviera, Decompositions and reductions of snarks, J. Graph Theory 22 (1996), no. 3, 253-279.

[13] E. Steffen, Classification and characterizations of snarks, Discrete Math. 188 (1998), no. 1-3, 183-203.

[14] P. G. Tait, On the colouring of maps, Proc. Roy. Soc. Edinburgh 10 (1880), 501-503. 
[15] P. G. Tait, Further remarks on the colouring of maps, Proc. Roy. Soc. Edinburgh 10 (1880), 728-729.

[16] J. J. Watkins, On the construction of snarks, Ars Combin. 16-B (1983), 111-123. 